\title{
Time-domain and frequency-domain modeling of nonlinear optical components at the circuit-level using a node-based approach
}

\author{
Martin Fiers ${ }^{1,4, *}$, Thomas Van Vaerenbergh ${ }^{1,4}$, Ken Caluwaerts ${ }^{2}$, Dries Vande \\ Ginste $^{3}$, Benjamin Schrauwen ${ }^{2}$, Joni Dambre ${ }^{2}$ and Peter Bienstman ${ }^{1,4}$ \\ ${ }^{1}$ Photonics Research Group (INTEC), Ghent University - imec, \\ Sint-Pietersnieuwstraat 41, B-9000 Ghent, Belgium \\ ${ }^{2}$ Electronics and Information Systems (ELIS), Ghent University, \\ Sint-Pietersnieuwstraat 41, B-9000 Ghent, Belgium \\ ${ }^{3}$ Electromagnetics Group (INTEC), Ghent University, \\ Sint-Pietersnieuwstraat 41, B-9000 Ghent, Belgium \\ ${ }^{4}$ Center for Nano- and Biophotonics (NB-Photonics), Ghent University, \\ Sint-Pietersnieuwstraat 41, B-9000 Ghent, Belgium \\ *Corresponding author: martin.fiers@intec.ugent.be
}

We present a tool that aids in the modeling of optical circuits, both in the frequency and in the time domain. The tool is based on the definition of a node, which can have both an instantaneous input-output relation, as well as different state variables (e.g. temperature and carrier density) and differential equations for these states. Furthermore, each node has access to part of its input history, allowing the creation of delay lines or digital filters. Additionally, a node can contain sub-nodes, allowing the creation of hierarchical networks. This tool can be used in numerous applications such as frequency-domain analysis of optical ring filters, time-domain analysis of optical amplifiers, microdisks and microcavities. Although we mainly use this tool to model optical circuits, it can also be used to model other classes of dynamical systems, such as electrical circuits and neural networks. (c) 2011 Optical Society of America

OCIS codes: $190.4390,220.4830,130.3120$ 


\section{Introduction}

There is a variety of tools available to simulate the behaviour of optical components. Many of these tools are limited to small networks of only a handful of components. To model these components or small networks, different techniques are used, such as Finite Difference Time Domain (FDTD) (e.g. MEEP [1,2]), eigenmode expansion, Time Domain Traveling Wave (TDTW) [3], Split Step Methods (SSM) [3] and Coupled Mode Theory (CMT). These tools mostly differ in complexity and in the level to which they contain physical details. FDTD, for example, is based on Maxwell's equations and therefore is a full-wave optical solver. The major drawback is that FDTD is computationally very expensive. On the other side, CMT is an approximate description, but extremely elegant and fast, only needing a few variables to describe a complex system. This is achieved by eliminating all spatial dependencies in the physical problem.

In this software landscape, there are tools to design complex optical circuits consisting of many components. For example, ASPIC [4] is used for calculating the steady-state response of optical circuits, and VPI [5] is mainly used to study the time-domain evolution. PicWAVE uses a time-domain travelling wave (TDTW) optical model [6], and RSoft Optsim uses SSM [7]. There are also approaches that use Modified Node Analysis (MNA), such as OptiSPICE $[8,9]$, which allows simulation of mixed electronical and optical circuits. All of these new tools will become indispensible in the future when designing and optimizing large optical circuits.

In this paper, we present a different node-based approach. The advantage of our approach is that both time and frequency domain can be investigated in the same framework, and that each component can be represented in a natural way using variables such as the optical

field, the temperature and the carrier density, without needing to be mapped on to voltage or current such as in the MNA approach. It uses only a small set of variables per component, similar to CMT, which means the simulations are extremely fast compared to other methods such as FDTD, TDTW and SSM, with the drawback of losing accuracy. Also, we provide a mechanism to eliminate instantaneous components from the network, reducing the amount of components we need to simulate in time domain. Our tool, named CAPHE [10] can also be used to simulate novel computational systems such as photonic reservoirs [11]. It is written in $\mathrm{C}++$ for optimal performance, with a Python front-end for ease of use and interfacing to a large collection of scientific libraries.

The rest of the paper is structured as follows: first we define a component with its basic properties such as the scatter matrix and the ODE equations. After that, we create a circuit consisting of several nodes, and we explain how we can derive the total scatter matrix from this. We then explain how scalable the software tool is, and how we can eliminate linear, instantaneous nodes from the circuit. After that we compare the accuracy of the simplified 
methods (used in this framework) with very accurate methods, and we conclude with an illustration of how eliminating nodes can speed up simulations considerably in the time domain.

\section{Model}

To design a complex circuit simulator, we first need to define the behaviour of one component. A node consists of $N$ ports, see Fig. 1. A linear instantaneous transmission between port $s_{i n, i}$ and $s_{\text {out }, j}$ is defined through the scatter matrix $\mathbf{S}_{i j}$. Two optional time-domain descriptions

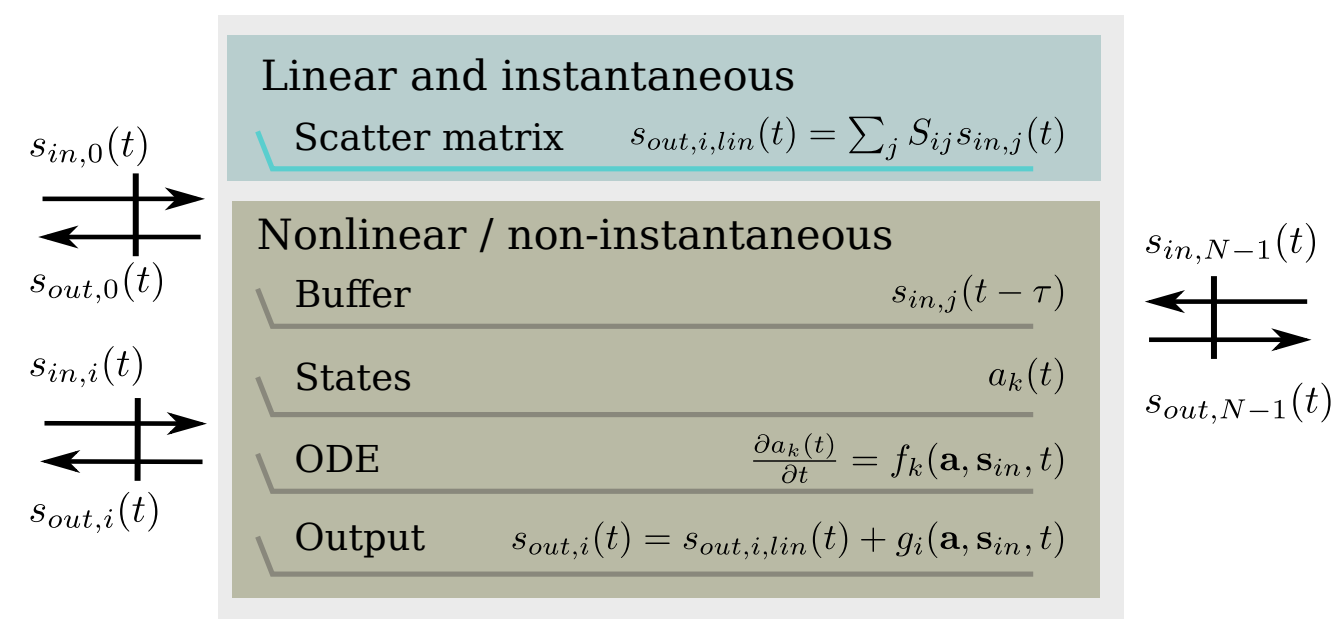

Fig. 1. Structure of a node with $\mathrm{N}$ ports. A linear and instantaneous node is only described by a scatter matrix $\mathbf{S}$. States (e.g. temperature and free carriers) can be added. In this case the node becomes nonlinear.

can be added to enrich this component (see Fig. 1, bottom): First, one can add a buffer to store the inputs $s_{i n, i}$ at previous timesteps. This can be used if one wishes to model a delayed waveguide or a digital filter. Second, we can add internal states to the node. This can be used to describe the rate equations of, e.g., a laser or the complex amplitude of a resonator. We use a set of ordinary differential equations $(O D E)$ to describe the component in terms of its internal variables. There is no restriction on the form of the equations, so highly nonlinear components can be easily modeled. Because of these two additions, the output $s_{\text {out }, i}$ is now a sum of the linear part and a term describing the nonlinear character of the component.

The main novelty of our framework is that the frequency-domain $\mathbf{S}$-matrix for components that only have a linear instantaneous transmission can be used to significantly speed up time-domain simulations of networks with both instantaneous and non-instantaneous components. In the next two sections, we give more insight into the frequency-and time-domain characteristics of these nodes. 


\section{A. Steady-state equations}

As previously mentioned, a linear instantaneous component can be described by a single scatter matrix $S$, and a linear input-output relation: $s_{\text {out }, i}(t)=\sum_{j} S_{i j} s_{i n, j}(t)$. Using this relationship we can describe all linear phenomena in the frequency domain. For example, a ring resonator can be modeled by combining a directional coupler and a waveguide. Both have a very simple scatter matrix, but when combined, they create resonances at certain frequencies. As these nodes contain no memory, we call them memoryless (ML) nodes.

\section{B. Time-domain equations}

We represent time-domain signals as complex amplitudes $s(t)$, modulating a carrier frequency $\omega_{c}$. The actual input at each port is then

$$
E(t)=s(t) e^{j \omega_{c} t}+c . c .
$$

Representing the signal by $s(t)$ is beneficial from a numerical point of view, as we can now integrate over $s(t)$ which varies much slower than $E(t)$. Obviously, as the bandwidth of the input signal increases, we will need more samples per time unit to correctly simulate the system.

For each component we can now optionally add time-domain equations which leads in its most general form to an input-output relation of the following form:

$$
s_{\text {out }, i}(t)=\sum_{j} \mathbf{S}_{i j} s_{i n, j}(t)+s_{\text {ext }, i}
$$

Here, $s_{e x t, i}$ is a generalized source term. E.g. for a continuous wave source, $s_{e x t}=A$, where $A$ is the complex amplitude of the source. For a waveguide with delay $\tau$, the simple relation $s_{\text {out }, i}(t)=s_{\text {ext }, i}=B s_{i n, 1-i}(t-\tau)$, for $i \in[0,1]$, holds. Here, $B$ is the complex value which determines the loss and phase change of the waveguide. Note that for this waveguide, there is no longer an instantaneous behaviour, i.e. $\mathbf{S}_{i j}$ is zero in (2).

Differential equations can be added to describe the evolution of some internal variables, e.g., temperature and free carriers in a laser, as a function of time and inputs.

As soon as there is a source term present in (2), the component is not instantaneous anymore. We call these nodes memory-containing (MC) nodes (Fig. 1, bottom), as opposed to the the memoryless (ML) nodes. Depending on whether the delays in a waveguide are important for a simulation, one can model them with delay (which makes it MC), or without delay (as a ML component), the latter having the advantage that we can eliminate it from the total network. This is explained in the next section. 


\section{Circuit scatter matrix}

We use the node from Fig. 1 as a basic building block to create an optical circuit. From this circuit, a total scatter matrix can be calculated in the frequency domain that describes the transmission to and from ports in the network. This matrix can become very big. Hence, in this section we also derive techniques to eliminate the ML nodes from the circuit. This is a crucial feature in our approach, which reduces the number of variables needed in the time domain, hence improving the simulation speed. To do this, we split the input/output vector $\mathbf{s}_{\text {in/out }}(t)$ into $\mathbf{s}_{i n / o u t, M C}(t)$ and $\mathbf{s}_{\text {in/out }, M L}(t)$, the input and output, respectively, to MC and ML nodes. For simplicity we will omit the time dependency in the following equations. The size of this s-vector equals the total number of ports in the circuit.

We can describe the way the different components are connected as follows:

$$
\left(\begin{array}{c}
\mathbf{s}_{i n, M C} \\
\mathbf{s}_{i n, M L}
\end{array}\right)=\mathbf{C}_{\text {tot }}\left(\begin{array}{c}
\mathbf{s}_{\text {out }, M C} \\
\mathbf{s}_{\text {out }, M L}
\end{array}\right)=\left(\begin{array}{ll}
\mathbf{C}_{M C, M C} & \mathbf{C}_{M C, M L} \\
\mathbf{C}_{M L, M C} & \mathbf{C}_{M L, M L}
\end{array}\right)\left(\begin{array}{l}
\mathbf{s}_{\text {out }, M C} \\
\mathbf{s}_{\text {out }, M L}
\end{array}\right) .
$$

Here, $\mathbf{C}_{t o t, i j}$ is a binary connection matrix which only contains a 1 if port $\mathrm{i}$ is connected to port $\mathbf{j}$. As a consequence, $\mathbf{C}_{t o t}$ is symmetric and contains at most 1 element per row and at most 1 element per column, with zeros on the diagonal.

The behaviour of each of the individual nodes can be described by the following equations:

$$
\begin{aligned}
\mathbf{s}_{\text {out }, M L} & =\mathbf{S}_{M L, M L} \mathbf{s}_{i n, M L} \\
\mathbf{s}_{\text {out }, M C} & =\mathbf{S}_{M C, M C} \mathbf{s}_{i n, M C}+\mathbf{s}_{e x t, M C},
\end{aligned}
$$

in which we define the scatter matrices $\mathbf{S}_{M L, M L}$ and $\mathbf{S}_{M C, M C}$. These are block diagnal matrices, with each block representing the scatter matrix from a ML resp. MC node. The

second term in equation (5), $\mathbf{s}_{e x t, M C}$, is the generalized source term described earlier, see also equation (2).

With all equations above we can derive the input at the active ports, given only $\mathbf{s}_{e x t, M C}$. This is done as follows: replace $\mathbf{s}_{o u t, M L}$ in equation (3) using (4). Solve this system for $\mathbf{s}_{i n, M C}$. This gives

$\mathbf{s}_{i n, M C}=\left(\mathbf{C}_{M C, M C}+\mathbf{C}_{M C, M L} \mathbf{S}_{M L, M L}\left(\mathbf{I}-\mathbf{C}_{M L, M L} \mathbf{S}_{M L, M L}\right)^{-1} \mathbf{C}_{M L, M C}\right) \mathbf{s}_{o u t, M C}=\mathbf{C s}_{\text {out }, M C}$

We then substitute (5) in the equation above. This yields

$$
\mathbf{s}_{i n, M C}=\left(\mathbf{I}-\mathbf{C S}_{M C, M C}\right)^{-1} \mathbf{C s}_{e x t}=\mathbf{S s}_{e x t}
$$

We have now successfully eliminated the memoryless nodes and end up with a smaller scatter matrix $\mathbf{S}$ of the network. 
Note that the matrix inversion in (6) is of a special type: $\mathbf{C}_{M L, M L}$ only permutes the elements of $\mathbf{S}_{M L, M L}$, and $\mathbf{S}_{M L, M L}$ is a block diagonal matrix. The resulting matrix $\mathbf{I}$ $\mathbf{C}_{M L, M L} \mathbf{S}_{M L, M L}$ is therefore sparse. However, the resulting matrix after inversion is not always sparse. This depends on the topology of the original network and on the individual scatter matrices of the ML nodes.

The final ingredient in our model are the internal states, which are stored in the total variable vector $\mathbf{a}(t)$.

$$
\frac{d \mathbf{a}(t)}{d t}=\mathbf{f}\left(\mathbf{a}, \mathbf{s}_{i n}, t\right)
$$

This ordinary differential equation (ODE) can now be solved easily as we can evaluate $\mathbf{f}$ as follows: at each timestep we loop over all MC to calculate $\mathbf{s}_{\text {ext }}$. Then we can calculate $\mathbf{s}_{i n}$ from equation (7). With this, we can evaluate $f_{k}\left(\mathbf{a}, \mathbf{s}_{i n}, t\right)=\frac{d a_{k}(t)}{d t}$.

\section{Optimizations in the frequency domain}

In equation (6) and (7) we need to solve a system of equations. For example, in equation (7) we need to solve

$$
\left(\mathbf{I}-\mathbf{C S}_{M C, M C}\right) \mathbf{X}=\mathbf{C}
$$

for $\mathbf{X}$. This can be done by first doing a LU factorization, followed by forward and backward substitution to find $\mathbf{X}$. A similar reasoning is done for the inversion in (6). Solving a system is almost always preferred above matrix inversion in terms of speed and stability. Optionally, since these matrices are sparse, we can use KLU to solve this system very efficiently [12-14].

To benchmark the speed-up, we consider the use case of a Coupled Resonator Optical Waveguide (CROW). A CROW is a sequence of optical rings, see Fig. 2. Each section is made of a directional coupler (with coupling values $\kappa_{i}$ ) and two waveguides, which then couples to the next section. This structure is used for creating optical filters.

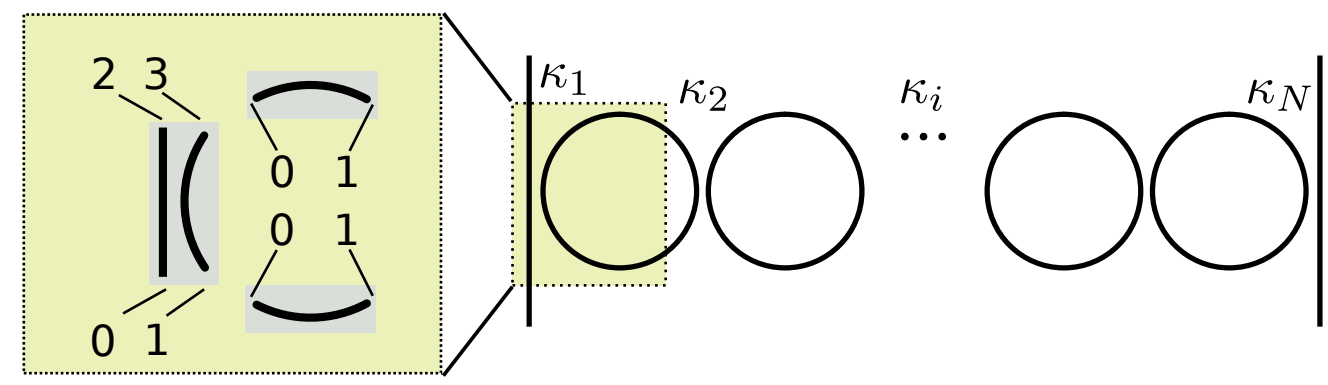

Fig. 2. A Coupled Resonator Optical Waveguide (CROW). Each section is subdivided in a directional coupler and two waveguides. Port numbers are shown in the left. 
The directional coupler and the waveguide are ML components with four resp. two ports. This means there are eight ports per CROW section. By increasing the number of sections, we find out what network size our framework can handle in the frequency domain. This is shown in Fig. 3, where we compare the time spent by different matrix strategies as a function of the ML ports. As can be seen in the figure, a large number of CROW sections can easily be handled. This proves the technique is useful for analyzing very complex systems in steady-state regime.

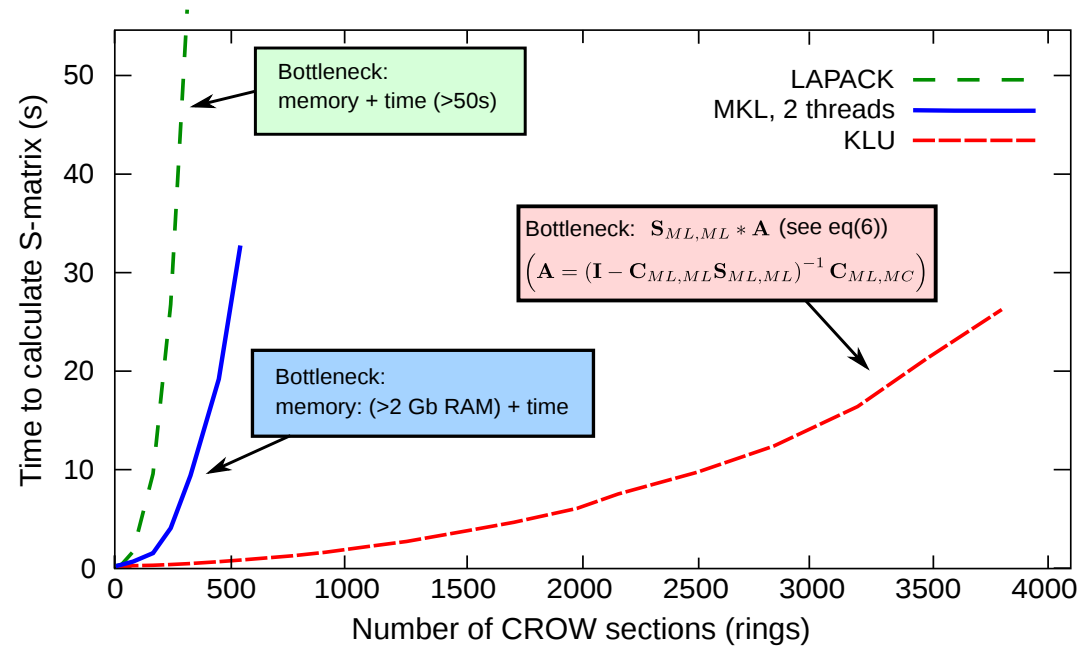

Fig. 3. Calculating the frequency response of a passive network. Using KLU, a sparse matrix solver suited for circuit matrices, we can easily calculate scatter matrices of very large networks.

\section{A. Robustness and accuracy in the time domain.}

This section describes two algorithms for integrating the ODE and compares the results to rigorous FDTD simulations.

The first integration scheme is a simple forward Euler with fixed time step $d t$, the second one is an advanced stepping routine based on Bulirch-Stoer [16]. The latter uses an adaptive stepsize, to guarantee accuracy and stability. As example network, we use a system of two coupled photonic crystal cavities. The model equations for this system are:

$$
\begin{aligned}
\frac{\mathrm{d} a_{j}}{\mathrm{~d} t} & =\left[i\left(\omega_{0}+\delta \omega_{j}\right)-\frac{1}{\tau}\right] a_{j}+d s_{j ; 0,+}+d s_{j ; 1,+}, \\
s_{j ; 1,-} & =\exp (i \phi) s_{j ; 0,+}+d a_{j}, \\
s_{j ; 0,-} & =\exp (i \phi) s_{l ; 1,+}+d a_{j},
\end{aligned}
$$




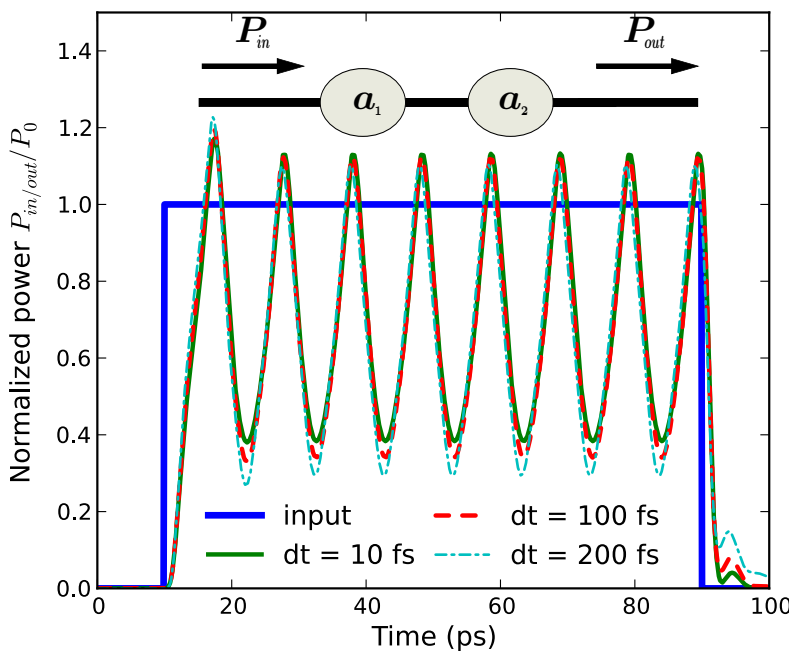

(a) Fixed steps

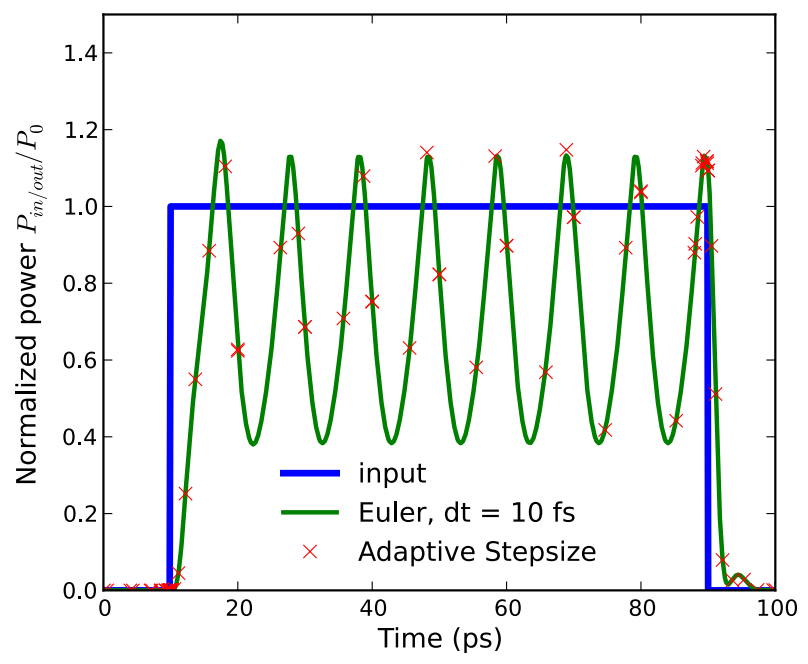

(b) Adaptive stepsize

Fig. 4. Two integration routines. Left: Forward Euler integration. With larger timesteps, the accuracy decreases. Right: Using adaptive stepsize and an advanced stepper routine, the solver automatically uses the optimal dt in order to maintain a desired accuracy (e.g. during switch on, the dt is reduced). Same parameters used as in [15].

for $j=1,2$. Here $d=i \exp (i \phi / 2) / \sqrt{\tau}$, where $\tau$ is the lifetime of the cavity and $\phi$ represents the phase that depends on the waveguide length and the resonator mirror reflection properties. The nonlinear frequency shift is $\delta \omega_{j}=-\left|a_{j}\right|^{2} /\left(P_{0} \tau^{2}\right)$, with $P_{0}$ the 'characteristic nonlinear power' of the cavity. In these equations $\left|a_{j}\right|^{2}$ is the energy in the cavity mode. $\left|s_{j ; k,+}\right|^{2}$ (resp. $\left|s_{j ; k,-}\right|^{2}$ ) represents the power flowing in (resp. out) port $k$ (for $k=0,1$ ) of cavity $j$ (for $j=1,2$ ). Port 1 of cavity 1 is connected with port 0 of cavity 2 . Thus, $\left|s_{1 ; 0,+}\right|^{2} \equiv P_{\text {in }}$ is the input power, $\left|s_{2 ; 1,-}\right|^{2} \equiv P_{\text {out }}$ is the transmitted power. We assume no input from the right, $s_{2 ; 1,+}=0$. This system will exhibit self-pulsation under certain assumptions, as studied in $[15]$.

In [15] we also showed that the waveforms from the simulation are almost identical to the waveforms from a full-wave FDTD simulation modeling the same system. The difference in simulation time motivates the use of this simulator: It takes 10 hours to simulate this oscillation in 2D FDTD, versus a few milliseconds with CMT, with only a slight sacrifice in accuracy. The equations governing this system are highly nonlinear, yet the numerics remain very stable. Furthermore, using adaptive stepsize, one is assured that the most important details of the simulation are taken into consideration, with an automatic choice of discretization steps. Whereas for a fixed stepsize algorithm, like forward Euler, accuracy over the whole 
simulation domain can only be obtained by choosing a very small stepsize (Fig. 4(b)), this is not the case for the adaptive stepsize algorithm: during switch on and switch off, there are a lot of discretization steps, while in between the adaptive stepsize solution can follow the reference solution with fewer discretization steps (Fig. 4(b)).

\section{Improving the simulation speed in the time domain}

If a network contains both ML and MC nodes, one can eliminate the ML nodes prior to starting the time domain simulation. The speed of the time domain simulation depends on the size of the scatter matrix after eliminating the ML nodes. We demonstrate this by modeling a large network of interconnected nodes. The topology is a regular 2D grid of Semiconductor Optical Amplifiers (SOA). Each SOA is connected to its neirest neighbours, in a structure called a swirl topology [17], see Fig. 5(a). To connect the SOAs, we used a combination of splitters and waveguides. We compare two systems. In the first system the ML nodes behave as MC nodes, in the second system we first eliminate all ML nodes. In the first case, we need to calculate the light propagation in each splitter and waveguide separately, which means the simulation will take longer, and consume more memory, as in the second case. This is illustrated in Fig. 5(b).

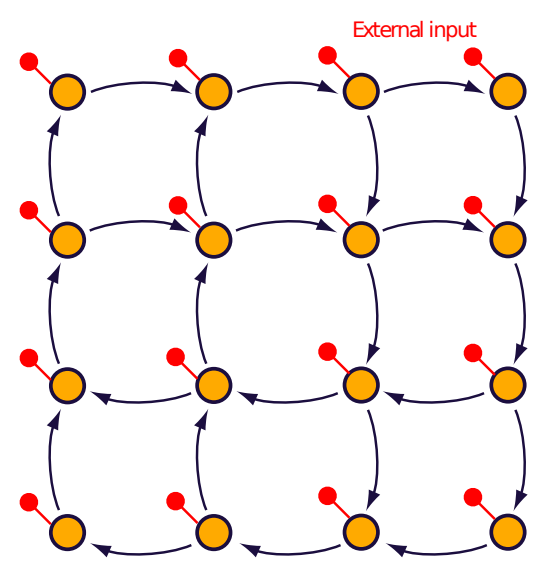

(a)
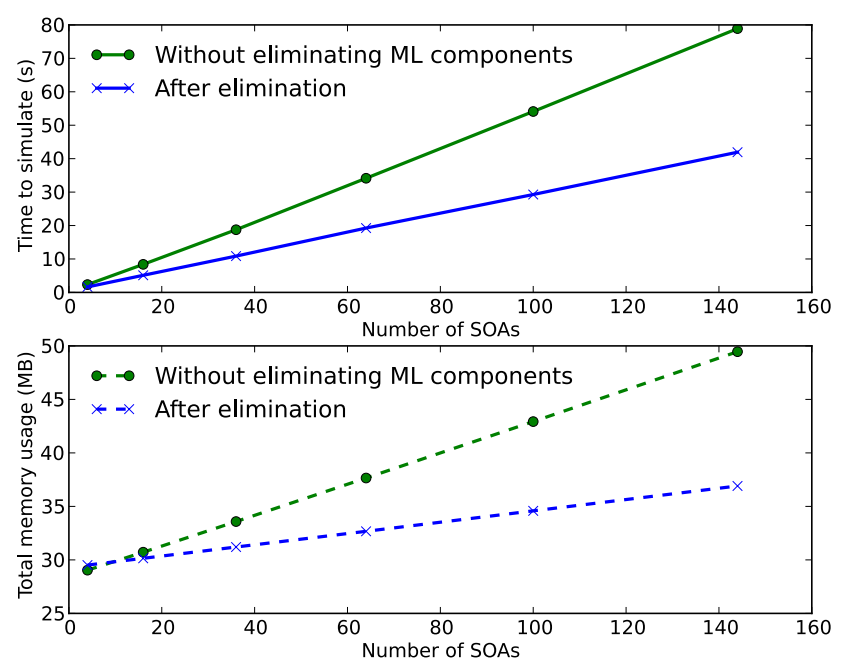

(b)

Fig. 5. Left: Topology used to simulate a complex system with ML and MC nodes. Each circle represents a SOA. Splitters are not shown. Right: The simulation time and memory usage increases linearly with the number of SOAs. Clearly there is an advantage by eliminating the ML nodes, both in terms of speed and memory usage. 
The total simulation time is mainly determined by the evaluation of the ODEs of the individual SOAs and the matrix multiplication from equation (7). There is a clear benefit of eliminating the ML nodes: the simulation speed is approximately halved as shown in Fig. 5(a) (top). The calculation time for evaluating the ODEs is the same for both systems. The memory usage is shown in the bottom graph of Fig. 5(a): It is a sum of the memory allocated in $\mathrm{C}++$ and in Python. The offset is due to initialization overhead in Python. For all simulations, we used a buffer that stores 500 timesteps. Because we eliminated the ML nodes, the memory requirements are greatly reduced. Since we use sparse matrices, calculation time and memory requirements scale linearly as a function of the network size.

\section{Conclusion}

In this paper, we demonstrated a framework that enables modeling of optical circuits both in the time and in the frequency domain. It is suited for calculating the steady state characteristics of very large networks, and to model highly nonlinear systems in the time domain after eliminating linear instantaneous components. By eliminating these components, we reduce the effective size of the network, and the time-domain simulation is speeded up. The tool is very general and the internal variables can be expressed naturally depending on the application domain, which makes it attractive for other dynamical systems such as electrical circuits and neural networks.

\section{Acknowledgements}

This work is supported by the interuniversity attraction pole (IAP) Photonics@be of the Belgian Science Policy Office and the ERC NaResCo Starting grant. M. Fiers acknowledges the Special Research Fund of Ghent University. T. Van Vaerenbergh and K. Caluwaerts are supported by the Flemish Research Foundation (FWO-Vlaanderen) for a PhD Grant.

\section{References}

1. A. F. Oskooi, D. Roundy, M. Ibanescu, P. Bermel, J. D. Joannopoulos, and S. G. Johnson, "MEEP: A flexible free-software package for electromagnetic simulations by the FDTD method," Computer Physics Communications 181, 687-702 (2010).

2. E. Lambert, M. Fiers, S. Nizamov, M. Tassaert, and W. Bogaerts, "Python bindings for the open source electromagnetic simulator meep," Computing in Science and Engineering (2010).

3. G. Agrawal, Nonlinear Fiber Optics, Third Edition (Optics and Photonics) (2007).

4. "http://www .aspicdesign.com/".

5. "http://www.vpiphotonics.com/optical_systems.php" .

6. "http://www.photond.com/products/picwave.htm" . 
7. "http://www.rsoftdesign. com/products.php?sub=System+and+Network\&itm= OptSim".

8. P. Gunupudi, T. Smy, J. Klein, and Z. Jakubczyk, "Self-consistent simulation of optoelectronic circuits using a modified nodal analysis formulation," Advanced Packaging, IEEE Transactions on 33, 979 -993 (2010).

9. T. Smy, P. Gunupudi, S. Mcgarry, and W. N. Ye, "Circuit-level transient simulation of configurable ring resonators using physical models," America 28, 1534-1543 (2011).

10. "http://photonics.intec. ugent. be/research/topics.asp?ID=138" .

11. K. Vandoorne, W. Dierckx, B. Schrauwen, D. Verstraeten, R. Baets, P. Bienstman, and J. Van Campenhout, "Toward optical signal processing using photonic reservoir computing," Optics Express 16, 11182-11192 (2008).

12. T. A. Davis and E. P. Natarajan, "Algorithm 8xx: Klu, a direct sparse solver for circuit simulation problems," .

13. T. A. Davis, Direct methods for sparse linear systems (2006).

14. K. Stanley, "Klu: a clark kent sparse lu factorization algorithm for circuit matrices." (2004 SIAM Conference on Parallel Processing for Scientific Computing (PP04), 2004).

15. B. Maes, M. Fiers, and P. Bienstman, "Self-pulsing and chaos in series of coupled nonlinear micro-cavities," Physical Review B11 7911 (200911).

16. W. H. Press, S. A. Teukolsky, W. T. Vetterling, and B. P. Flannery, "Numerical recipes: The art of scientific computing," (2007).

17. K. Vandoorne, J. Dambre, D. Verstraeten, B. Schrauwen, and P. Bienstman, "Parallel reservoir computing using optical amplifiers," IEEE Transactions on Neural Networks 22, 1469-1481 (2011). 